\title{
Osteossarcoma: experiência do Serviço de Oncologia Pediátrica da Santa Casa de Misericórdia de São Paulo*
}

\author{
Osteosarcoma: experience of the Pediatric Oncology clinic \\ of the Santa Casa de Misericórdia Hospital in São Paulo
}

Helaine Cristina de Castro ${ }^{1}$, Karina de Cássia Braga Ribeiro², Paula Bruniera ${ }^{3}$

\section{RESUMO}

Objetivos: Descrever as características clínico-laboratoriais das crianças e adolescentes com osteossarcoma acompanhadas no Serviço de Oncologia Pediátrica da Santa Casa de Misericórdia de São Paulo e determinar as taxas de sobrevida livre de evento e sobrevida global, correlacionando-as com os principais fatores prognósticos. Métodos: Estudo retrospectivo realizado no Serviço de Oncologia Pediátrica da Santa Casa de Misericórdia de São Paulo compreendendo o período entre abril de 1991 e setembro de 2002. Nesse período foram diagnosticados 60 casos de osteossarcoma. Observaram-se

Trabalho realizado no Serviço de Oncologia Pediátrica do Departamento de Pediatria da Santa Casa de Misericórdia de São Paulo - FCMSCMSP - São Paulo (SP), Brasil.

1. Médica Assistente do Departamento de Pediatria da Santa Casa de Misericórdia de São Paulo, Mestra em Pediatria pela Faculdade de Ciências Médicas da Santa Casa de Misericórdia de São Paulo - FCMSCMSP - São Paulo (SP), Brasil.

2. Doutora, Professora Assistente do Departamento de Medicina Social da Faculdade de Ciências Médicas da Santa Casa de Misericórdia de São Paulo - FCMSCMSP - São Paulo (SP), Brasil.

3. Doutora, Professora Assistente do Departamento de Pediatria da Faculdade de Ciências Médicas da Santa Casa de Misericórdia de São Paulo, Chefe do Serviço de Oncologia e Hematologia Pediátrica do Departamento de Pediatria da Santa Casa de Misericórdia de São Paulo - FCMSCMSP - São Paulo (SP), Brasil.

Endereço para correspondência: Helaine Cristina de Castro, Rua Mônaco, 120, Ap. 93-B, Jardim Têxtil - 03413-030 - São Paulo (SP), Brasil. Tel.: (11) 9121-8246; fax: (11) 6192-5770.

E-mail: helainecc@hotmail.com

Recebido em 9/11/05. Aprovado para publicação em 17/4/08.

Copyright RBO2008 predomínio do sexo masculino $(61,7 \%)$, raça nãobranca $(65 \%)$ e idade superior a 10 anos $(70 \%)$; história de trauma prévio em $53,3 \%$, relato de dor em $95 \%$ e aumento de volume em $80 \%$ dos casos. $O$ joelho foi a região mais comprometida (74,9\%). Em 70,7\% dos casos o tumor apresentava tamanho inferior a 1/3 do comprimento do osso afetado, em $25 \%$ o nível sérico de desidrogenase láctica era igual ou maior que 500U/L e em 48,3\% o nível de fosfatase alcalina era igual ou maior que $1.000 \mathrm{U} / \mathrm{L}, 25 \%$ dos pacientes apresentavam metástase pulmonar visível ao diagnóstico e $53,3 \%$ pertenciam ao subtipo osteoblástico. $O$ tratamento quimioterápico pré e pós-operatório foi realizado em $\mathbf{7 8 , 3 \%}$ dos pacientes, $64 \%$ foram submetidos a procedimento cirúrgico conservador, $68,1 \%$ apresentaram necrose tumoral graus I e II e houve recidiva em $60 \%$ dos casos. Resultados: Com seguimento de 48 meses observaram-se sobrevida livre de doença em $28,6 \%$ dos pacientes e sobrevida global de 38,4\%. Conclusões: Os autores concluem que o diagnóstico precoce é fundamental, assim como novos estudos são necessários para aprimorar o manuseio e o tratamento desses pacientes e melhorar a sobrevida.

$$
\begin{gathered}
\text { Descritores - Osteossarcoma; Sobrevida; Prognóstico; } \\
\text { Criança; Estudos retrospectivos }
\end{gathered}
$$

\section{ABSTRACT}

Objectives: To describe clinical and laboratorial features of children and adolescents with osteosarcoma seen at the Pediatric Oncology clinic of the Santa Casa de Misericórdia Hospital in São Paulo and to 
determine the uneventful rates of survival and the overall survival, and correlate such rates to the main factors of prognosis. Methods: Retrospective study carried out at the Pediatric Oncology clinic of the Santa Casa de Misericórdia Hospital in São Paulo from April 1991 to September 2002. 60 cases of osteosarcoma were identified in the period. The authors saw a predominance of the male sex (61.7\%), non-white race (65\%), and age above 10 years $(70 \%)$; history of previous trauma in $53.3 \%$, reporting pain in $95 \%$, and volume increase in $80 \%$ of the cases. The knee joint was the involved region in most cases (74.9\%). In 70.7\% of the cases, the tumor was smaller than 1/3 of the bone affected, in $25 \%$ the serum lactic dehydrogenase level was equal to or greater than $500 U / L$, and in $48.3 \%$, the alkaline phosphatase level was equal to or greater than $1000 \mathrm{U} / \mathrm{L}, 25 \%$ of the patients presented with lung metastasis that was visible in the diagnosis, and $53.3 \%$ were of the osteoblastic subtype. Pre and post surgical chemotherapy was conducted in $78.3 \%$ of the patients, $64 \%$ were submitted to conservative surgery, $68.1 \%$ had tumoral necrosis grades I and II, and recurrence was seen in $60 \%$ of the cases. Results: With a 48 month follow-up, they observed disease-free survival in $28.6 \%$ of the patients, and overall survival in $38.4 \%$. Conclusions: They concluded that early diagnosis is a must, and that new studies are required to improve the management and treatment of this patients and to improve survival.

Keywords - Osteosarcoma; Survivorship (Public Health); Prognosis; Child; Retrospective studies

\section{INTRODUÇÃO}

O osteossarcoma é a mais freqüente neoplasia óssea maligna da infância e adolescência. Ocorre nas duas primeiras décadas da vida, principalmente no pico de estirão do crescimento na puberdade; é a terceira neoplasia mais freqüente nesta década da vida. O prognóstico está relacionado com diversas variáveis, tais como local do tumor primário, tamanho inicial, existência ou não de metástases ao diagnóstico, sexo, idade, alterações citogenéticas, subtipo histológico e resposta ao tratamento quimioterápico pré-operatório. Nas últimas décadas, com a realização de quimioterapia adjuvante e neo-adjuvante, a taxa de sobrevida e a qualidade de vida têm melhorado sobremaneira ${ }^{(1-6)}$.

A Santa Casa de São Paulo é um hospital pioneiro no tratamento de tumores ósseos e tem participado ativamente do processo de evolução diagnóstica e terapêutica dessa neoplasia. Inicialmente, o tratamento era apenas paliativo; o aprimoramento da avaliação diagnóstica e das técnicas cirúrgicas juntamente com o advento da quimioterapia permitiu a preservação e funcionalidade do membro na maioria dos casos e melhora significativa na sobrevida desses pacientes. No início dos anos 90, o Grupo de Oncologia Ortopédica, juntamente com o Serviço de Oncologia Pediátrica, passou a integrar o Grupo Brasileiro Cooperativo para o Tratamento de Tumores Ósseos na Infância (GBCTTOI), que preconiza o uso de quimioterapia pré-operatória.

Este estudo tem por objetivo relatar a experiência do Serviço no manuseio e tratamento dos pacientes com osteossarcoma, descrevendo suas principais características, contribuindo para melhor conhecimento do comportamento dessa neoplasia na população estudada.

\section{MÉTODOS}

Trata-se de estudo retrospectivo que foi realizado no Serviço de Oncologia do Departamento de Pediatria da Santa Casa de Misericórdia de São Paulo, considerando os casos de osteossarcoma diagnosticados em crianças e adolescentes no período entre abril de 1991 e setembro de 2002. Avaliamos os prontuários e exames de imagem dos pacientes e observamos a evolução dos mesmos até junho de 2004. Este trabalho foi aprovado pela Comissão Científica do Departamento de Pediatria e pelo Comitê de Ética em Pesquisa em Seres Humanos da Irmandade da Santa Casa de Misericórdia de São Paulo.

$\mathrm{Na}$ avaliação foram observados: sexo, idade $(<\mathrm{ou} \geq$ de 10 anos), raça (branca e não-branca), tempo de história, presença de dor e aumento de volume, relação com trauma local prévio, local acometido pelo tumor, tamanho do tumor $(<$ ou $\geq 1 / 3$ do comprimento do osso acometido), existência de fratura patológica, presença de metástase ao diagnóstico, nível sérico de de- 
sidrogenase láctica (DHL $<\mathrm{e} \geq 500 \mathrm{U} / \mathrm{L}$ ) e fosfatase alcalina (FA $<\mathrm{e} \geq 1.000 \mathrm{U} / \mathrm{L}$ ), subtipo histológico, tipo de abordagem cirúrgica, resposta histológica do tumor à quimioterapia neo-adjuvante (grau de necrose) e ocorrência de recaída.

Durante o período deste estudo, foram utilizados cinco protocolos seqüenciais para o tratamento do osteossarcoma, preconizando quimioterapia neo-adjuvante e adjuvante com cirurgia. Os esquemas utilizados foram: I) de 1991 a 1992: protocolo composto por cisplatina e doxorrubicina; II) de 1993 a 1996, estudo III do GBCTTOI composto por carboplatina intra-arterial, ifosfamida, epidoxorrubicina, carboplatina intravenosa e metotrexato em dose alta; III) de 1997 a 1998, estudo IV do GBCTTOI composto por cisplatina, carboplatina, doxorrubicina, ifosfamida; IV) de 1998 a 2000, protocolo com ifosfamida, doxorrubicina, cisplatina; e V) de 2001 a 2002, protocolo Osteossarcoma 2000 do GBCTTOI composto por cisplatina, doxorrubicina, ifosfamida e ciclofosfamida.

A análise estatística descritiva para as variáveis quantitativas foi realizada por meio da observação dos valores mínimos e máximos, cálculo de médias, desviopadrão e medianas; para as variáveis qualitativas foram calculadas as frequiências absolutas e relativas. A análise da sobrevida livre de evento (SLE) e da sobrevida global (SG) foi realizada pelo método de Kaplan-Meier. Esses testes estatísticos foram realizados através do programa Statistical Package for Social Sciences (SPSS) versão 12.0 .

Nesse período foram diagnosticados 60 casos de osteossarcoma em pacientes com idade variando de cinco a 16 anos e mediana de 11 anos, sendo $70 \%$ com idade igual ou superior a 10 anos; $61,7 \%$ eram do sexo masculino e a razão entre os sexos foi de $1: 1,6 ; 65 \%$ pertenciam a outras raças que não a branca.

Em relação aos sinais e sintomas iniciais, observamos que 53,3\% referiram trauma prévio na região afetada pela neoplasia, 95,0\% relatavam dor no local, $80,0 \%$ apresentavam aumento de volume local e 11,7\% possuíam fratura patológica. $\mathrm{O}$ tempo médio entre o início destes e o diagnóstico definitivo foi de 104 dias, com mediana de 65 dias, mínimo de um e máximo de 1.058 dias.
Todos os pacientes apresentavam comprometimento do esqueleto apendicular, sendo $51,7 \%$ no terço distal do fêmur, $23,2 \%$ no terço proximal da tíbia, $10,0 \%$ no terço proximal do úmero, $6,7 \%$ no terço proximal do fêmur, $5,0 \%$ no terço médio do fêmur, $1,7 \%$ no terço distal da tíbia e $1,7 \%$ no terço distal da fíbula. Dos 60 pacientes foi possível rever $41(68,3 \%)$ radiografias simples do local primário prévias ao início do tratamento; observamos que a neoplasia primária apresentou tamanho igual ou maior a um terço do segmento ósseo comprometido em 12 (29,3\%) pacientes e foi menor em 29 (70,7\%).

Entre os pacientes, $25 \%$ apresentavam metástase pulmonar ao diagnóstico; destes, em três havia metástases concomitantes em outros locais.

Ao diagnóstico, o nível sérico da DHL foi dosado em 51 pacientes, destes $39,2 \%$ apresentavam valor igual ou superior a 500U/L; e o de FA em 58 pacientes, sendo o nível sérico igual ou maior a 1.000 U/L em 48,3\%.

Com relação ao subtipo histológico, observamos em $53,3 \%$ ser osteoblástico, em 20,0\% condroblástico, em 8,3\% fibroblástico, em 5,0\% telangiectásico, em 1,7\% de pequenas células; em 11,7\% o subtipo não foi determinado.

Dos pacientes, 56 (93,3\%) foram submetidos à ressecção cirúrgica, três abandonaram o tratamento e um faleceu antes do momento preconizado para esta intervenção. Das crianças elegíveis para a cirurgia, seis $(10,7 \%)$ foram submetidas à amputação sem quimioterapia neo-adjuvante e $50(89,3 \%)$ receberam de um a seis ciclos de quimioterapia pré-operatória. Destas, 19 (38,0\%) foram submetidas à cirurgia ablativa devido à progressão do tumor e $31(62,0 \%)$ à cirurgia conservadora: uma $(3,2 \%)$ giroplastia de Van Ness, 21 $(67,7 \%)$ endopróteses não-convencionais e nove $(29,1 \%)$ reconstruções biológicas. Apenas três pacientes apresentaram comprometimento da margem cirúrgica e todos haviam sido submetidos a cirurgia conservadora.

A resposta do tumor à quimioterapia foi avaliada, de acordo com a classificação de grau de necrose proposta por Huvos et al ${ }^{(2)}$, em 47 (94,0\%) casos. Destes, $40,4 \%$ apresentaram grau I, 27,7\% grau II, $12,8 \%$ grau III e $19,1 \%$ grau IV. 


\section{RESULTADOS}

Houve recidiva da neoplasia em $60,0 \%$ dos pacientes; o pulmão foi o principal sítio de recaída $(88,9 \%)$, seguido do local primário $(47,2 \%)$. Observamos que, com seguimento de 48 meses, $25 \%$ dos pacientes estavam vivos e sem sinais de doença, $50 \%$ falecidos por progressão da neoplasia, 8,3\% mortos por outras causas e $16,7 \%$ não retornaram ao serviço, sendo considerados como perdidos de seguimento.
Em relação às taxas de sobrevida (tabela 1), observamos diferença estatisticamente significativa apenas em relação ao sexo feminino para SLE (gráfico 1) e aos níveis séricos elevados de FA (gráfico 2) e DHL (gráfico 3) e à recidiva (gráfico 4) para SG. Quando ao grau de necrose, observamos diferença, porém estatisticamente não significativa (gráficos 5 e 6). A SLE e a SG em 48 meses foram de $28,6 \%$ e $38,5 \%$, respectivamente (gráficos 7 e 8).

TABELA 1

Características da casuística estudada e respectivas sobrevida livre de evento e sobrevida global em 48 meses de acordo com as diversas variáveis. Serviço de Oncologia Pediátrica da Santa Casa de São Paulo, 1991 a 2002

\begin{tabular}{llllclc}
\hline \multicolumn{1}{c}{ Variável (N) } & Categoria & N (\%) & SLE-\% & p & SG-\% & p \\
\hline Idade & $<10$ anos & $18(30,0)$ & 35,1 & 0,283 & 60,9 & 0,081 \\
(60) & $\geq 10$ anos & $42(70,0)$ & 25,8 & & 29,4 & \\
Sexo & F & $24(40,0)$ & 16,8 & 0,046 & 35,1 & 0,556 \\
(60) & M & $36(60,0)$ & 35,9 & & 40,8 & \\
Raça & Branca & $21(35,0)$ & 21,3 & 0,501 & 35,4 & 0,563 \\
(60) & Não branca & $39(65,0)$ & 31,8 & & 39,5 & \\
Tamanho & $<1 / 3$ & $29(70,7)$ & 34,8 & 0,526 & 32,8 & 0,433 \\
(41) & $\geq 1 / 3$ & $12(29,3)$ & 31,4 & & 47,7 & \\
Metástase & Sim & $15(25,0)$ & 10,3 & 0,267 & 20,8 & 0,229 \\
(60) & Não & $45(75,0)$ & 33,1 & & 42,6 & \\
FA (U/L) & $<1.000$ & $47(81,0)$ & 30,9 & 0,993 & 44,5 & 0,047 \\
(58) & $\geq 1.000$ & $11(19,0)$ & 26,5 & & 27,3 & \\
DHL (U/L) & $<500$ & $31(60,8)$ & 43,2 & 0,073 & 56,9 & 0,003 \\
(51) & $\geq 500$ & $20(39,2)$ & 18,2 & & 15,8 & \\
Grau de necrose & I e II & $32(68,1)$ & 18,5 & 0,107 & 34,6 & 0,158 \\
(47) & III e IV & $15(31,9)$ & 46,4 & & 57,1 & \\
Recidiva & Sim & $36(60,0)$ & & & 20,8 & 0,0007 \\
& Não & $24(40,0)$ & & & 72,8 & \\
\hline
\end{tabular}

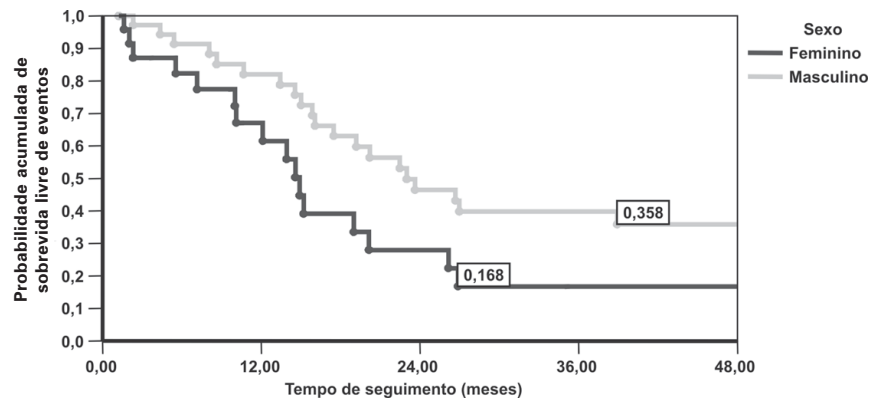

Gráfico 1 - Curva de sobrevida livre de evento para sexo dos 60 portadores de osteossarcoma. Serviço de Oncologia Pediátrica do Departamento de Pediatria da Santa Casa de São Paulo, 1991 a 2002.

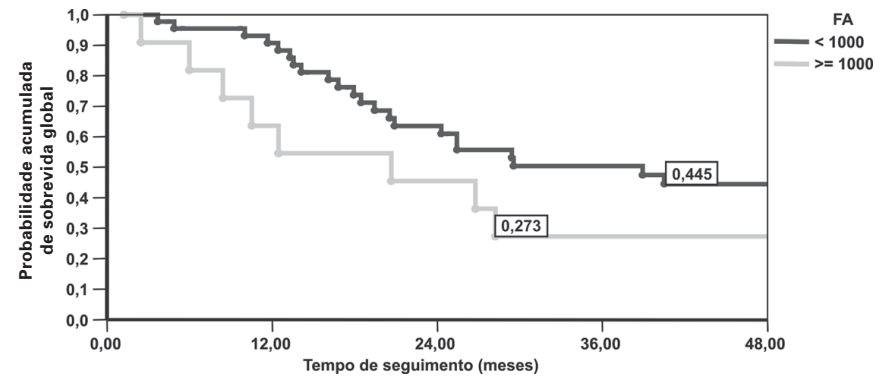

Gráfico 2 - Curva de sobrevida global para nível sérico de FA ao diagnóstico de 58 portadores de osteossarcoma. Serviço de Oncologia Pediátrica do Departamento de Pediatria da Santa Casa de São Paulo, 1991 a 2002. 


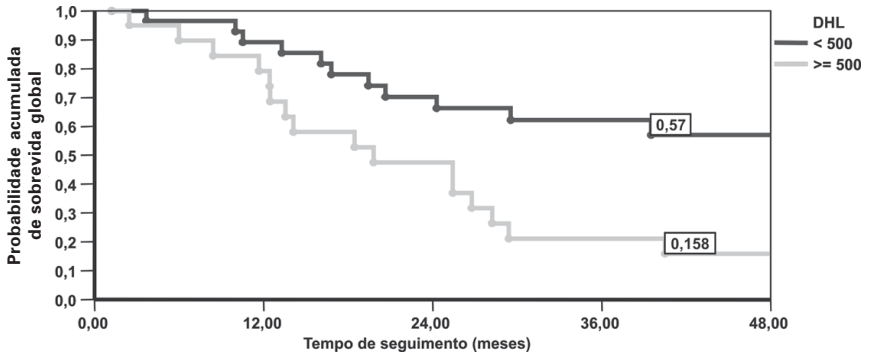

Gráfico 3 - Curva de sobrevida global para nível sérico de DHL ao diagnóstico de 51 portadores de osteossarcoma. Serviço de Oncologia Pediátrica do Departamento de Pediatria da Santa Casa de São Paulo, 1991 a 2002.

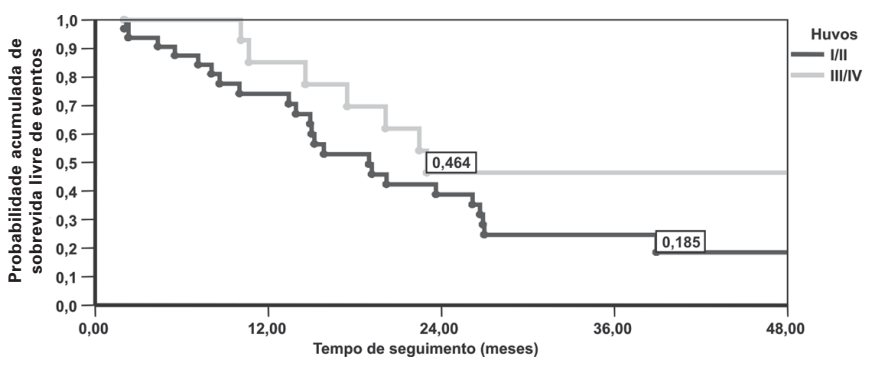

Gráfico 5 - Curva de sobrevida livre de evento para o grau de necrose causado pela quimioterapia dos 60 portadores de osteossarcoma. Serviço de Oncologia Pediátrica do Departamento de Pediatria da Santa Casa de São Paulo, 1991 a 2002.

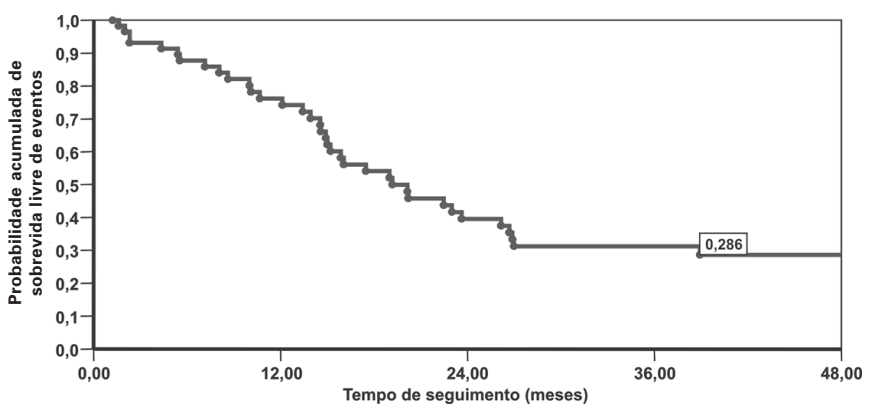

Gráfico 7 - Curva de sobrevida livre de evento

\section{DISCUSSÃO}

O osteossarcoma foi a segunda neoplasia $(11,4 \%)$ mais freqüente no Serviço de Oncologia do Departamento de Pediatria da Santa Casa de Misericórdia de São Paulo; isso difere da incidência mundial, na qual esta é a sexta neoplasia mais frequiente ${ }^{(4)}$. Provavelmente, esse fato se deve ao grande número de pacien-

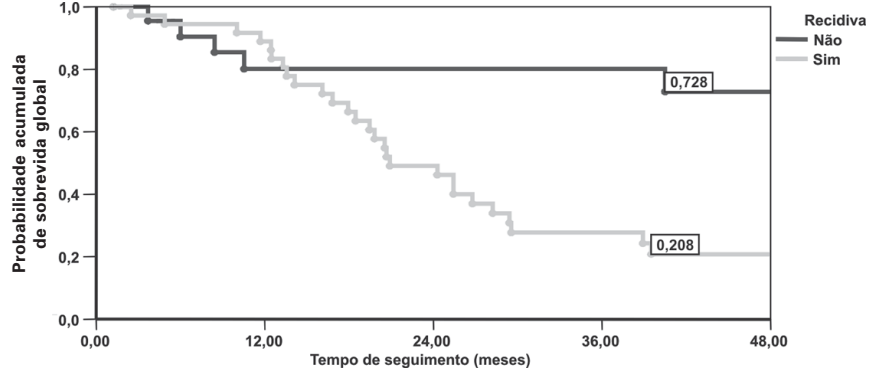

Gráfico 4 - Curva de sobrevida global para a ocorrência de recidiva da neoplasia dos 60 portadores de osteossarcoma. Serviço de Oncologia Pediátrica do Departamento de Pediatria da Santa Casa de São Paulo, 1991 a 2002.

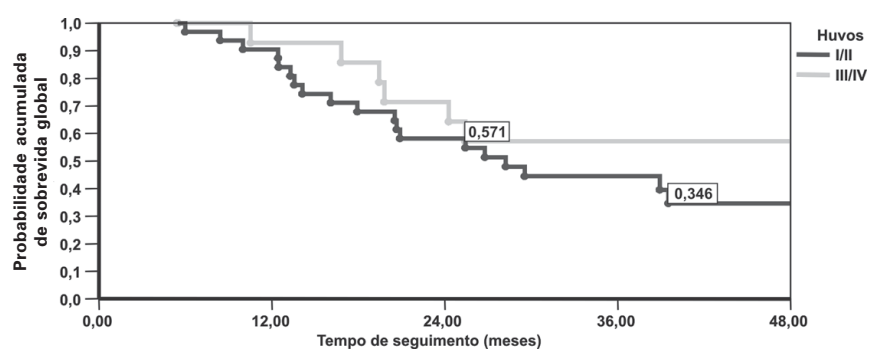

Gráfico 6 - Curva de sobrevida global para o grau de necrose causado pela quimioterapia dos 60 portadores de osteossarcoma. Serviço de Oncologia Pediátrica do Departamento de Pediatria da Santa Casa de São Paulo, 1991 a 2002.

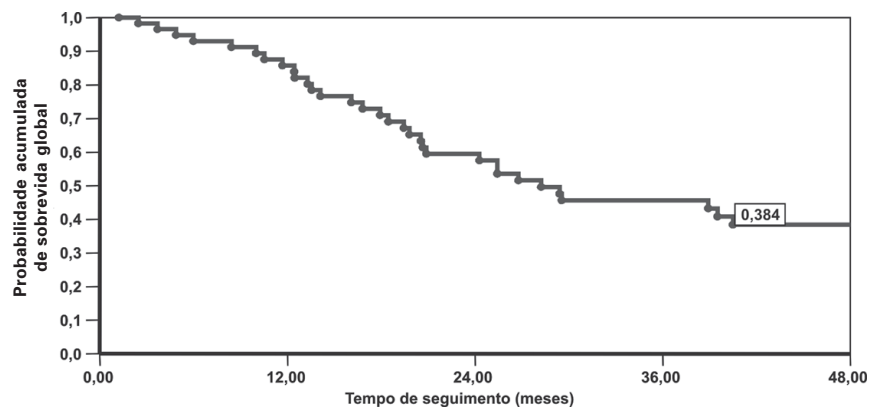

Gráfico 8 - Curva de sobrevida global

tes atendidos no Departamento de Ortopedia e Traumatologia.

Neste estudo, assim como na literatura, houve predomínio do sexo masculino, incidência maior na segunda década da vida, com pico na fase do estirão de crescimento para ambos os $\operatorname{sexos}^{(4,6-8)}$. Nesta casuística observamos predomínio da raça não-branca; este 
fato se deve provavelmente à grande miscigenação ocorrida no nosso país, o que dificulta a caracterização do grupo racial ao qual pertence cada indivíduo.

O intervalo de tempo entre os primeiros sinais e sintomas (dor, aumento de volume, fratura) e a realização do diagnóstico definitivo foi prolongado (104 dias); isso pode ser explicado pelo fato de o Serviço prestar atendimento a população de baixo nível socioeconômico-cultural e com dificuldade no acesso ao serviço de saúde. Acresce-se a isso o fato de esta ser uma doença relativamente rara, com a qual muitos profissionais não estão familiarizados e, conseqüentemente, não a consideram como possibilidade diagnóstica. Queremos salientar que, na fase inicial, sua identificação pode ser realmente difícil, pois os sinais e sintomas são inespecíficos e as imagens radiográficas podem ser normais; portanto, o seguimento cuidadoso de crianças e adolescentes que apresentam dor óssea sem diagnóstico estabelecido é de extrema importância para que o diagnóstico seja feito o mais breve possível. Muitas vezes, as crianças e seus responsáveis relacionam a dor a um trauma sofrido previamente; entretanto, essa relação é subjetiva, sugerindo ser esse o fator responsável pela dor, tanto para o paciente quanto para o profissional que o atendeu, mascarando o fator etiológico e atrasando o diagnóstico. Algumas vezes, o acaso colabora com o paciente e a ocorrência de fratura patológica pode antecipar o diagnóstico.

Os locais mais freqüentemente comprometidos foram as regiões próximas às placas de crescimento, onde há maior atividade óssea - mais freqüentemente o fêmur e a tíbia, principalmente na região do joelho - como tem sido citado, ao longo dos anos, na literatura ${ }^{(4,7-10)}$.

O tamanho do tumor primário ao diagnóstico é fator prognóstico importante. Existem diversos estudos correlacionando essa característica à resistência das células neoplásicas aos agentes quimioterápicos e mostrando que, quanto maior o tumor, menor o grau de resposta à quimioterapia. Entretanto, não há um consenso sobre qual a melhor forma ou método para mensurar o tamanho do tumor primário; na literatura há descrições de medidas do comprimento, do volume, da percentagem e do diâmetro do tumor ${ }^{(7,11-13)}$. Optamos por considerar o tamanho do tumor em relação ao comprimento do osso envolvido medido através da radiografia simples do membro, pois dessa forma incluiríamos maior número de pacientes na análise; observamos que, por esse método, 29,3\% dos pacientes apresentaram tumor considerado grande $(\geq 1 / 3$ do comprimento do segmento ósseo).

Observamos $25 \%$ de pacientes metastáticos ao diagnóstico, valor superior ao descrito na literatura internacional $(15,0 \% \text { a } 20,0 \%)^{(4-5,14)}$. Entretanto, nossos dados são similares aos encontrados na literatura nacional ${ }^{(10)}$, que relatam até $38,0 \%$. Esse fato pode ser explicado pelo diagnóstico mais tardio na nossa população, o que favorece a progressão da doença.

Em relação ao subtipo histológico, a distribuição encontrada em nosso trabalho não foi diferente da observada na literatura, com predomínio do osteossarcoma osteoblástico $^{(4,7-8,15-17)}$.

Os níveis séricos da DHL e da FA nos casos avaliáveis estavam aumentados em 39,2\% e 48,3\% dos pacientes, respectivamente. Estudos ${ }^{(4,18)}$ têm mostrado que essas enzimas refletem a extensão da neoplasia no momento do diagnóstico, pois estão relacionadas à atividade celular e, quanto maior a disseminação das células neoplásicas, maior o nível sérico das mesmas.

Durante o período deste estudo foram utilizados cinco protocolos sequienciais para o tratamento do osteossarcoma, multicêntricos e de acordo com os estudos do GBCTTOI. Como os protocolos possuíam braços, o número de pacientes alocados em cada um foi pequeno, não sendo possível fazer análise comparativa com valor estatístico significativo.

A quimioterapia neo-adjuvante teve papel importante, permitindo número cada vez maior de intervenções conservadoras. Nesse grupo, a primeira cirurgia conservadora realizada foi a giroplastia de Van Ness, porém, apesar de ter apresentado boa funcionalidade, o resultado estético foi ruim. Também foram realizadas nove reconstruções biológicas e 21 substituições por endoprótese não convencional. Chamamos a atenção para o fato de que somente três pacientes apresentaram comprometimento da margem cirúrgica, mostrando a importância da quimioterapia no controle da neoplasia, permitindo ressecções cada vez menos extensas; destes, dois apresentaram somente recidiva local e 
estão vivos sem doença, e um apresentou doença refratária ao tratamento. Na literatura mundial podemos observar que os procedimentos conservadores tornaram-se cada vez mais freqüentes devido à eficácia do tratamento neo-adjuvante e a sobrevida atinge 70$85 \%(2,9,17,19-20)$.

Observamos que 31,9\% dos pacientes obtiveram boa resposta ao tratamento com graus de necrose III e IV, enquanto que $68,1 \%$ apresentaram graus de necrose I e II. Na literatura verificamos que a intensificação progressiva dos protocolos de tratamento promoveu impacto positivo no grau de necrose tumoral, atingindo $70-75 \%$ de bons respondedores, porém, os protocolos mais agressivos submetem os pacientes a riscos maiores devido à toxicidade aguda e efeitos colaterais tardios dos quimioterápicos, fazendo-se necessários maiores cuidados de suporte e manuseio ${ }^{(13,18,21-23)}$.

Apesar dos diferentes esquemas terapêuticos utilizados, avaliamos as SLE $(28,6 \%)$ e SG $(38,4 \%)$ deste

\section{REFERÊNCIAS}

1. Mckenna RJ, Schwinn CP, Soong KY, Higinbotham NL. Osteogenic sarcoma arising in Paget's disease. Cancer. 1964; 17:42-66.

2. Huvos AG, Rosen G, Marcove RC. Primary osteogenic sarcoma: pathologic aspects in 20 patients after treatment with chemotherapy en bloc resection, and prosthetic bone replacement. Arch Pathol Lab Med. 1977;101(1):14-8.

3. Schajowicz F. Tumores formadores de tejido óseo. In: Schajowicz F, editor. Tumores y lesiones seudotumorales de huesos y articulaciones. Buenos Aires: Editorial Panamericana; 1982. p. 36-119.

4. Link MP, Eilber F. Osteossarcoma. In: Pizzo PA, Poplack DG, editors. Principles and practice of pediatric oncology. $3^{\mathrm{a}}$ ed. Philadelphia: Lippincott - Raven Publishers; 1997. p. 889-920.

5. Meyers PA, Gorlick R. Osteosarcoma. Pediatr Clin North Am. 1997;44(4):973-89. Review.

6. Latorre MRDO, Franco EL. Epidemiologia dos sarcomas. Acta Oncol Bras. 1998;18(1):3-13.

7. Petrilli AS. Osteossarcoma - quimioterapia intra-arterial e fatores prognósticos [tese]. São Paulo: Escola Paulista de Medicina; 1988.

8. Próspero JD. Neoplasias produtoras de tecido ósseo. In: Prospero JD, editor. Tumores ósseos. São Paulo: Roca; 2001. p.1-43.

9. Petrilli AS, Epelman S, Mendes WL, Machado TMS, Brunetto M, Pereira W, et al. Osteosarcoma 2000: Preliminary Results of the Brazilian Cooperative Group. In: Thirty-Ninth Annual grupo e observamos resultados semelhantes aos da literatura nacional ${ }^{(9-10,20,24)}$ e diferentes dos da literatura internacional, que mostra índices superiores, variando entre 50 e $85 \%$. É muito importante identificar os fatores prognósticos do osteossarcoma para que possamos desenvolver terapias mais adequadas e que proporcionem maior sobrevida com melhor qualidade de vida para as crianças e adolescentes portadoras desta neoplasia.

\section{CONCLUSÃO}

Observamos que foram fatores prognósticos desfavoráveis, com diferença estatisticamente significativa, o sexo feminino para SLE; os níveis séricos elevados de FA e DHL e a recidiva para SG. E também concluímos que o diagnóstico precoce é fundamental, assim como novos estudos são necessários para aprimorar o manuseio e o tratamento desses pacientes e melhorar a sobrevida.
Meeting of the American Society of Clinical Oncology, 2003, Chicago, IL. ASCO, 2003. v. 22. p. 801-801.

10. Rech A, Castro Júnior CG, Mattei J, Gregianin L, Di Leone L, David A, et al. Características clínicas do osteossarcoma na infância e sua influência no prognóstico. J Pediatr (Rio J). 2004; 80(1):65-70.

11. Petrilli AS, Gentil FC, Epelman S, Lopes LF, Bianchi A, Lopes A, et al. Increased survival, limb preservation, and prognostic factors for osteosarcoma. Cancer. 1991;68(4):733-7.

12. Bacci G, Ferrari S, Bertoni F, Picci P, Bacchini P, Longhi A, et al. Histologic response of high-grade nonmetastatic osteosarcoma of the extremity to chemotherapy. Clin Orthop Relat Res. 2001;(386):186-96.

13. Bielack SS, Kempf-Bielack B, Delling G, Exner GU, Flege S, Helmke K, et al. Prognostic factors in high-grade osteosarcoma of the extremities or trunk: an analysis of 1,702 patients treated on neoadjuvant cooperative osteosarcoma study group protocols. J Clin Oncol. 2002;20(3):776-90.

14. Kager L, Zoubek A, Pötschger U, Kastner U, Flege S, KempfBielack B, Branscheid D, Kotz R, Salzer-Kuntschik M, Winkelmann W, Jundt G, Kabisch H, Reichardt P, Jürgens H, Gadner H, Bielack SS; Cooperative German-Austrian-Swiss Osteosarcoma Study Group. Primary metastatic osteosarcoma: presentation and outcome of patients treated on neoadjuvant Cooperative Osteosarcoma Study Group protocols. J Clin Oncol. 2003;21(10):2011-8. Comment in: J Clin Oncol. 2004; 22(24):5017; author reply 5017-8. 
15. Schajowicz F, Sissons HA, Sobin LH. The World Health Organization's histologic classification of bone tumors. A commentary on the second edition. Cancer. 1995;75(5):1208-14.

16. Hauben EI, Weeden S, Pringle J, Van Marck EA, Hogendoorn PC. Does the histological subtype of high-grade central osteosarcoma influence the response to treatment with chemotherapy and does it affect overall survival? A study on 570 patients of two consecutive trials of the European Osteosarcoma Intergroup. Eur J Cancer. 2002;38(9):1218-25. Comment in: Eur J Cancer. 2003;39(4):548-9.

17. Bacci G, Bertoni F, Longhi A, Ferrari S, Forni C, Biagini R, et al. Neoadjuvant chemotherapy for high-grade central osteosarcoma of the extremity. Histologic response to preoperative chemotherapy correlates with histologic subtype of the tumor. Cancer. 2003;97(12): 3068-75.

18. Bacci G, Picci P, Ferrari S, Orlandi M, Ruggieri P, Casadei R, at al. Prognostic significance of serum alkaline phosphatase measurements in patients with osteosarcoma treated with adjuvant or neoadjuvant chemotherapy. Cancer. 1993;71(4):1224-30.

19. Meskens M, Burssens A, Hoogmartens M, Fabry G. Osteogenic sarcoma in children. A retrospective study of 58 cases. Acta Orthop Belg. 1993;59(1):64-8.
20. Garcia RJ, Consentino E, Camargo OP, Batista PPR, Croci AT, Korukian M, et al. Tratamento ortopédico do osteossarcoma: Grupo Cooperativo Brasileiro de Tratamento do Osteossarcoma. Rev Bras Ortop. 1996;31(11):871-8.

21. Feugeas O, Guriec N, Babin-Boilletot A, Marcellin L, Simon P, Babin S, et al. Loss of heterozygosity of the RB gene is a poor prognostic factor in patients with osteosarcoma. J Clin Oncol. 1996;14(2):467-72. Erratum in: J Clin Oncol 1996;14(8):2411.

22. Bacci G, Ferrari S, Bertoni F, Ruggieri P, Picci P, Longhi A, et al. Long-term outcome for patients with nonmetastatic osteosarcoma of the extremity treated at the istituto ortopedico rizzoli according to the istituto ortopedico rizzoli/ osteosarcoma-2 protocol: an updated report. J Clin Oncol. 2000;18(24):4016-27.

23. Pakos EE, Ioannidis JP. The association of P-glycoprotein with response to chemotherapy and clinical outcome in patients with osteosarcoma. A meta-analysis. Cancer. 2003;98(3):581-9.

24. Penna V, Lopes A, Tanaka MH, Hu TC; Melaragno R; Epelman S. Osteossarcoma: tratamento multidisciplinar. Rev Bras Ortop. 1993;28(11/12):791-4. 